\title{
Intrathecal delivery of human ESC-derived mesenchymal stem cell spheres promotes recovery of a primate multiple sclerosis model
}

\author{
Li Yan', Bin Jiang ${ }^{1}$, Yuyu Niu², Hongxuan Wang ${ }^{1,3}$, Enqin Li ${ }^{1}$, Yaping Yan², Huiyan Sun ${ }^{1,4}$, Yanchao Duan², \\ Shaohui Chang' ${ }^{2}$, Guokai Chen', Weizhi $\mathrm{Ji}^{2}$, Ren-He Xu' and Wei Si ${ }^{2}$
}

\begin{abstract}
Nonhuman primate experimental autoimmune encephalomyelitis (EAE) is a valuable model for multiple sclerosis, an inflammatory demyelinating disease in the central nervous system (CNS). Human embryonic stem cell-derived mesenchymal stem cells (EMSC) are effective in treating murine EAE. Yet, it remains unknown whether the EMSC efficacy is translatable to humans. Here we induced a primate EAE model in cynomolgus monkeys and delivered EMSC in spheres $\left(E M S C_{s p}\right)$ to preserve the cell viability during long-distance transportation. EMSC $_{\mathrm{sp}}$ intrathecally injected into the CNS, remarkably reduced the clinical symptoms, brain lesions, and neuronal demyelination in the EAE monkeys during a 3-month observation. Whereas, symptoms in the vehicle control-injected EAE monkey remained and reduced slowly and MRI lesions in brain expanded. Moreover, EMSC could transdifferentiate into neural cells in vivo in the CNS

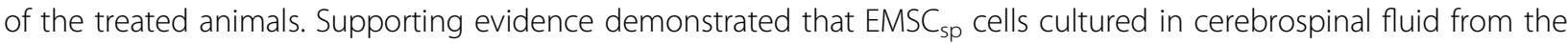
EAE monkeys largely converted to neural cells with elevated expression of genes for neuronal markers, neurotrophic factors, and neuronal myelination. Thus, this study demonstrates that EMSC $\mathrm{sp}_{\mathrm{s}}$ injected directly into the CNS, can attenuate the disease progression in the primate EAE model, highly encouraging for clinical translation.
\end{abstract}

\section{Introduction}

Multiple sclerosis (MS) is a chronic inflammatory disease of the central nervous system (CNS) featured by demyelination of neural fibers and malfunction of the neural signal transmission with high prevalence in Caucasians and females. The processes involve contributions of autoreactive $\mathrm{T}$ cells and autoantibodies that attack myelin, oligodendrocytes, and underlying nerves, resulting

\footnotetext{
Correspondence: Weizhi Ji (wji@lpbr.cn) or Ren-He Xu (renhexu@umac.mo) or Wei Si (siw@lpbr.cn)

${ }^{1}$ Faculty of Health Sciences, University of Macau, Taipa, Macau, China

${ }^{2}$ Yunnan Key Laboratory of Primate Biomedical Research, Institute of Primate

Translational Medicine, Kunming University of Science and Technology, Kunming, Yunnan, China

Full list of author information is available at the end of the article.

These authors contributed equally: Li Yan, Bin Jiang, Yuyu Niu

Edited by A. Rufini
}

in demyelination of neural fibers in the CNS and followed by axonal loss and neuronal damage ${ }^{1-4}$. Experimental autoimmune encephalomyelitis (EAE) has been widely used for studying the pathologic mechanisms and testing novel therapies for $\mathrm{MS}^{5}$. As the immune system in nonhuman primates (NHP) is similar to humans, EAE has been induced in NHP marmoset, rhesus, and cynomolgus monkeys to recapitulate the immunological and pathological processes and test the efficacy of novel therapies before clinical applications in $\mathrm{MS}^{6-9}$.

Stem cell therapies based on mesenchymal stem cells (MSCs) have been found effective for EAE in animal models ${ }^{10-16}$ and clinical trials on MS patients ${ }^{11,17-21}$ due to the dual properties - immunomodulation and neuroprotection $^{11,22-24}$. MSC are traditionally derived from a

\section{(c) 2018 The Author(s)}

(c) (i) Open Access This article is licensed under a Creative Commons Attribution 4.0 International License, which permits use, sharing, adaptation, distribution and reproduction cc in any medium or format, as long as you give appropriate credit to the original author(s) and the source, provide a link to the Creative Commons license, and indicate if changes were made. The images or other third party material in this article are included in the article's Creative Commons license, unless indicated otherwise in a credit line to the material. If material is not included in the article's Creative Commons license and your intended use is not permitted by statutory regulation or exceeds the permitted use, you will need to obtain permission directly from the copyright holder. To view a copy of this license, visit http://creativecommons.org/licenses/by/4.0/. 
variety of fetal and adult tissues ${ }^{25}$. We have previously reported two novel methods to efficiently differentiate human embryonic stem cells (hESCs) into MSC $(\text { EMSC })^{26,27}$, and EMSC can treat EAE in mice with superior therapeutic efficacy ${ }^{14}$. Compared with fetal and adult tissues, hESC possess several obvious advantages for MSC derivation including unlimited self-renewal, easiness for quality control, and fewer quality variations among different batches ${ }^{28,29}$. Thus, EMSC are highly suitable for development into a clinical-grade therapy and scalable for pipeline production. Recently, we found that spheroidal formation allows ambient storage of MSC for up to 10 days, which may replace the traditional cryopreservation method for cell transportation worldwide ${ }^{30}$.

Encouraged by these findings, we decided to test the EMSC efficacy on EAE induced in cynomolgus monkeys to promote the translation of EMSC as a therapy from mice to humans. Moreover, we hoped to test whether EMSC spheres $\left(\mathrm{EMSC}_{\mathrm{sp}}\right)$, transported under ambient conditions, are still effective for treatment of the NHP model.

\section{Results \\ EAE induction in monkeys \\ Immunization and symptom onset}

Eight female cynomolgus monkeys were used in this study (Fig. 1a). Seven of them (C1-C7) were immunized with an emulsion of myelin oligodendrocyte glycoprotein peptide $\left(\mathrm{MOG}_{35-55}\right)$ and complete Freund's adjuvant and $\mathrm{C} 8$ was a normal control. Symptoms were first observed in monkey (C1) at d19 including loss of appetite and general reduction of motor activities with the clinical score as 1.0. The disease score increased to 2.5 at $\mathrm{d} 21$ for the appearance of visual problem and left-sided hemiparesis. Three days later, the symptoms gradually relieved and maintained at score 0.5 (Fig. 1a and Fig. S1Aa). The symptomless $\mathrm{C} 2$ was left as a control for $\mathrm{C} 1$ for EMSC in vivo distribution assay.

Second immunization was given to the rest symptomless animals $\mathrm{C} 3-\mathrm{C} 7$ at $\mathrm{d} 33$. C4 exhibited obvious tremor of lower limbs at $\mathrm{d} 43$ and $\mathrm{C} 5$ developed visual problem and tremor of hind limbs at d44, which were both scored 1.0. Then incomplete paralysis of the hind limbs and right front limb developed in C5 at d47 (scored 2.75) (Fig. 1a and Figs. S1 Ab and c). Finally, third immunization was given to $\mathrm{C} 3, \mathrm{C} 6$, and $\mathrm{C} 7$ at $\mathrm{d} 77$, and only $\mathrm{C} 6$ manifested symptoms (tremor of the hind limbs, scored 1.0) at d146 (Fig. 1a and Fig. S1Ad). C3 and C7 displayed no clinical symptom after the three immunizations. Thus, the disease scores demonstrated pronounced inter-individual variations in the onset time and symptom severity.

\section{Lesions detected in the CNS via MRI}

Upon the symptom onset in $\mathrm{C} 1$, magnetic resonance imaging (MRI) was conducted on the brain of the monkey
(Fig. 1b). Focal hyper-intense regions in T2-weighted images were found in the left frontal lobe and cerebellum in $\mathrm{C} 1$ at $\mathrm{d} 20$. After the symptom onset, diffuse and cloudy hyper-intense lesions in both sides of the temporal lobe (only the left side is shown) and a small round lesion in the right frontal lobe were detected in $\mathrm{C} 4$ at $\mathrm{d} 49$ (Fig. 1b). In the brain of $\mathrm{C} 5$, intense expanding lesions were found at $\mathrm{d} 49$ in the frontal, temporal, and occipital lobes across both the white matter and gray matter with signs of swelling. MRI also detected lesions in the right temporal lobe and left occipital lobe in C6 on d119, however, symptoms started in this animal at d146. Although no obvious symptoms were observed on the animals C2, C3, and $\mathrm{C} 7$, lesions at different sizes were detected in various brain regions at $\mathrm{d} 119$ (Fig. 1b). These results suggest that great variations existed among the immunized monkeys in terms of the MRI lesion, onset time, and severity of symptoms, and the size and location of lesions in the CNS. Pathological processes occurred in all the seven immunized monkeys although symptoms were manifested in only four of them. If the MRI-detected brain lesions are considered as a sign of disease onset, the EAE induction rate is $100 \%$.

\section{Histological evidence for $E A E$}

To confirm the lesions in the CNS of the EAE-induced monkeys, we sacrificed $\mathrm{C} 1$ and $\mathrm{C} 8$ on $\mathrm{d} 32$. Luxol fast blue (LFB) staining revealed demyelination in the white matter of the frontal lobe of the brain and spinal cord of the EAE monkey $\mathrm{C} 1$ (Fig. 2Aa and b). Immunostaining for myelin binding protein (MBP) and oligodendrocytes on $\mathrm{C} 1$ displayed unilateral and subacute demyelination in both the white matter and gray matter in the right frontal lobe of its brain and spinal cord (Figs. 2Ac, d, and S1B). In addition, scattered $\mathrm{SMA}^{+}$cells indicated the damage of the blood-brain barrier (BBB) in lesioned areas compared with integral $\mathrm{BBB}$ in the brain of $\mathrm{C} 8$ (Fig. $2 \mathrm{Ba}$ ). $\mathrm{CD}^{+}$ cells, indicative of lymphocyte infiltration, were observed in the white matter of the brain of $\mathrm{C} 1$ (Fig. 2Bb).

\section{Rapid elimination of EMSC following i.v. injection into the monkeys}

As we needed to transport EMSC for a long distance from Macau to Kunming, we decided to test whether spheropreserved $\mathrm{EMSC}^{30}$ are effective in the monkey EAE model. First, $\mathrm{EMSC}_{\mathrm{sp}}$ derived from the $\mathrm{GFP}^{+}$Envy hESC line $^{31}$ were stored under ambient conditions for 3 days $\left(\mathrm{EMSC}_{\mathrm{sp}^{-} \mathrm{AC}-\mathrm{d} 3}\right)$ and transported by flight from the laboratory in Macau to the animal facility in Kunming on d4 (EMSC sp-AC-d4 $\left.^{-}\right)$(Fig. S2A). Before injection, the cell viability was verified over $95 \%$ (Fig. S2Ba).

To determine the best route of EMSC delivery into the animals, we first tried intravenous (i.v.) injection. Single cells $\left(1 \times 10^{7}\right.$ cells $\left./ \mathrm{kg}\right)$ dissociated from $\mathrm{EMSC}_{\mathrm{sp}^{-}} \mathrm{AC}-\mathrm{d} 4$ 


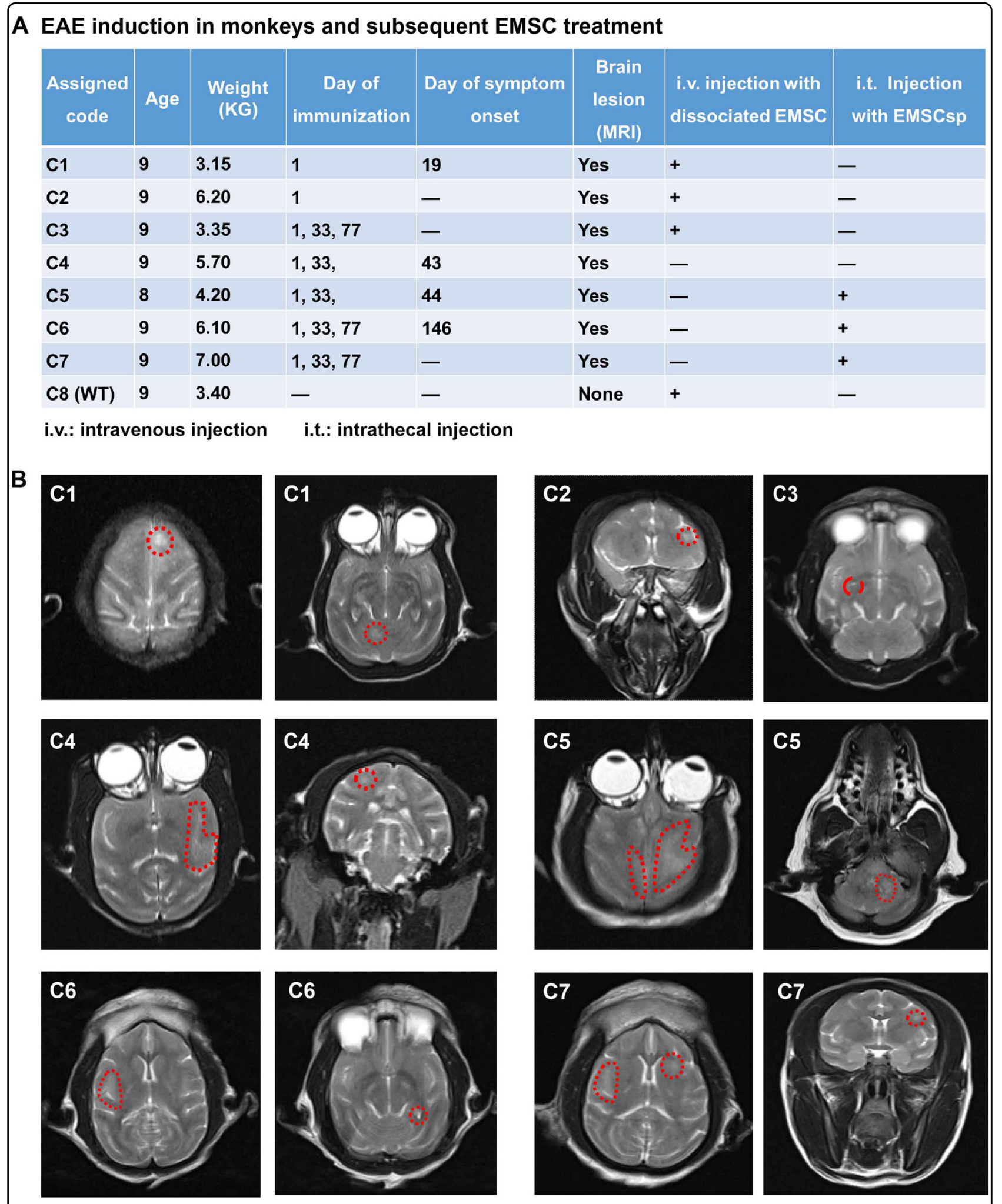

Fig. 1 EAE induction, EMSC treatment, and cerebral lesions in cynomolgus monkeys. a EAE induction in monkeys and subsequent EMSC treatment. $\mathbf{b}$ MRI images of all the EAE-induced monkeys. Displayed are representative images of T2-weighted lesions marked with red dotted lines 


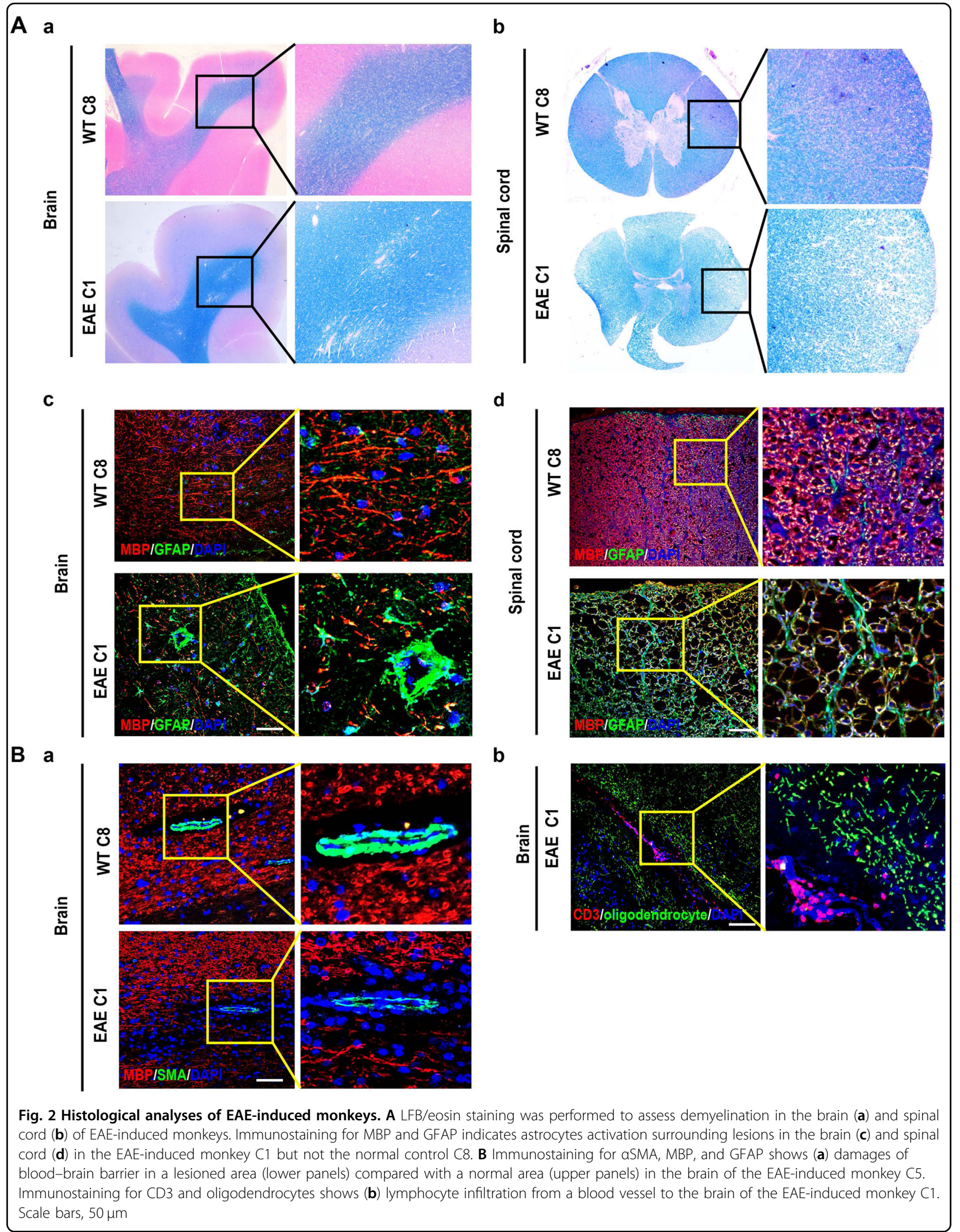


were i.v. infused into the monkeys ( $\mathrm{C} 1$ and $\mathrm{C} 8$ ) (Fig. S2Bb). The clinical score did not change and no side effect was observed on both $\mathrm{C} 1$ (Fig. S1Aa) and C8 for 4 days after the cell infusion. To investigate the cell distribution in vivo, $\mathrm{C} 1$ and $\mathrm{C} 8$ were sacrificed 4 days after the cell infusion. GFP DNA of the Envy EMSC was measured via quantitative PCR (qPCR) in each organ. The results showed that GFP DNA was detectable in almost all tissues in $\mathrm{C} 1$ and $\mathrm{C} 8$. Interestingly, the concentration of GFP DNA in EAE monkey $\mathrm{C} 1$ was about fourfold higher than that in normal control C8 (Fig. S2Ca and b).

As EAE monkeys $\mathrm{C} 2$ and $\mathrm{C} 3$ had lesions in the brain, but no obvious symptoms (Fig. 1a). C2 and C3 were used to study the distribution and retention time of transplanted EMSC in the animals at earlier and later time points. We administrated the green fluorescent protein positive $\left(\mathrm{GFP}^{+}\right)$EMSC (via i.v. injection with $1 \times 10^{7}$ cells $/ \mathrm{kg}$ ) into $\mathrm{C} 2$ and $\mathrm{C} 3$ (Fig. 2B and S2Bb). The two animals were sacrificed at 1 and 7 days post the cell injection, respectively. Low concentrations of GFP DNA were detected in $\mathrm{C} 2$ and $\mathrm{C} 3$ (Fig. S2Cc and d). However, $11 \mathrm{ng} / \mu \mathrm{g}$ gDNA in the spinal cord of $\mathrm{C} 3$ is about ninefold lower than that in $\mathrm{C} 1$ at $\mathrm{d} 4$ (Fig. S2Cd). These results suggest that (1) EMSC, following i.v. injection, distributed to all the isolated tissues, in which the spinal cord had the highest concentration of EMSC especially in the EAE monkeys; (2) EMSC declined in all the tested tissues from $\mathrm{d} 4$ to $\mathrm{d} 7$, and no obvious symptom improvement was observed in $\mathrm{C} 1$ in short time window.

\section{Symptoms in EAE-induced monkeys relieved following intrathecal injection of $\mathrm{EMSC}_{\mathrm{sp}}$}

The above observations led us to seek more efficient methods for cell delivery. As administration of MSC spheres via intracerebroventricular injection reduces chronic alcohol intake in rat $^{32}$, we reasoned that direct delivery of small $\mathrm{EMSC}_{\mathrm{sp}}$ via intrathecal injection (i.t.) may allow the cells to exempt from biological challenges that EMSC encounter via i.v. injection. Thus, we generated $\mathrm{GFP}^{+} \mathrm{EMSC}_{\mathrm{sp}}$ at $1.5 \times 10^{4}$ cells/sphere and about $200 \mu \mathrm{m}$ in diameter ${ }^{30}$. EMSC $_{\text {sp-AC-d4 }}$ (equivalent of $2 \times 10^{7}$ EMSC) were i.t. injected into three EAE monkeys C5, C6, and C7. Phosphate-buffered saline (PBS) was injected i.t. into EAE monkey $\mathrm{C} 4$ as a vehicle control (Fig. 3a). Clinical scores, peripheral blood and cerebrospinal fluid (CSF), MRI, and histological analyses were conducted on the animals.

\section{Clinical scores}

Upon symptom onset, C5 had sharply increased score that reached around 2.5 during $\mathrm{d} 47-\mathrm{d} 52$. Following the i.t. injection of $\mathrm{EMSC}_{\mathrm{sp}}$ at $\mathrm{d} 52$, its symptoms rapidly alleviated within 3 days (Videos S1 and S2) and then completely disappeared within 1 week, and no side effects such as hyperspasmia and fever were observed (Fig. S1Ac). Half month later (d68), some symptoms reoccurred on C5 with mild tremor of the right forelimb, apathy, and reduced movement and appetite, which was scored 1.0 (Fig. S1Ac). We administered C5 via i.t. injection of $\mathrm{EMSC}_{\mathrm{sp}}$ for the second time at d74 (Fig. 4a). Similar to the first time, the symptoms on C5 relieved within 3 days (Videos S3 and S4), its appetite recovered and response to stimulation was swift again. However, the symptoms relapsed (scored 0.5-1.2) and relieved (scored 0) twice after the second injection (Fig. S1Ac). Thus, we performed the third i.t. injection of $\mathrm{EMSC}_{\mathrm{sp}}$ on C5 at d113 (Fig. 3a). The symptoms disappeared within 44 days without relapse (Fig. S1Ac).

The i.t. injection of $\mathrm{EMSC}_{\mathrm{sp}}$ was performed on the EAE monkey C6 at d152 (Fig. 4a). The symptoms on C6 started on $\mathrm{d} 145$ and completely recovered after the first $\mathrm{EMSC}_{\mathrm{sp}}$ injection (Fig. S1Ad). Similar to the treatment course of C5, repeat treatments were administrated to C6 to secure the efficacy. Two more $\mathrm{EMSC}_{\mathrm{sp}}$ treatments were given to C6 at d184 and d215, respectively. During the 3-month treatment course, we did not see any symptom relapse. We also conducted i.t. injection of $\mathrm{EMSC}_{\mathrm{sp}}$ three times on the EAE-induced monkey $\mathrm{C} 7$ who had only brain lesions (Fig. 1) but no symptoms (Fig. 1a and Fig. 3a). No side effect or symptoms were observed in $\mathrm{C} 7$ during the entire experiment. Compared with the three EAE-induced monkeys treated with $\mathrm{EMSC}_{\mathrm{sp}}$, the symptoms of the vehicle control $\mathrm{C} 4$ had no alleviation following the PBS injection and lasted for a total of 58 days, disappeared for 11 days, and then relapsed on $\mathrm{d} 113$ to score 0.5 (Fig. S1Ab).

Thus, the three i.t. injections were performed on C5, C6, and C7 (Fig. 3a). The injection dates are not identical among the three monkeys, due to the different starting times of their symptoms. These results suggest that $\mathrm{EMSC}_{\mathrm{sp}}$ delivered via i.t. injection into the CNS of the EAE monkeys alleviated the clinical symptoms of the animals without causing obvious side effects during and after the $\mathrm{EMSC}_{\mathrm{sp}}$ treatments for $>3$ months.

\section{Assays of peripheral blood and CSF}

To assess the general immune responses during the disease progression and EMSC administration in the EAE monkeys, we counted cells in their peripheral blood and detected cytokine levels in their CSF during the entire process of the experiments. As shown in Fig. S3, the numbers of white blood cells and neutrophils remarkably increased after the onset of the disease in EAE monkeys. Four days after i.v. infusion of EMSC, the numbers of neutrophils and lymphocytes slightly changed in both the control C8 and C1 (Fig. S3A). However, no specific tendency of white blood cells was observed in C5-C7, compared with C4 without treatment (Fig. S3B). 


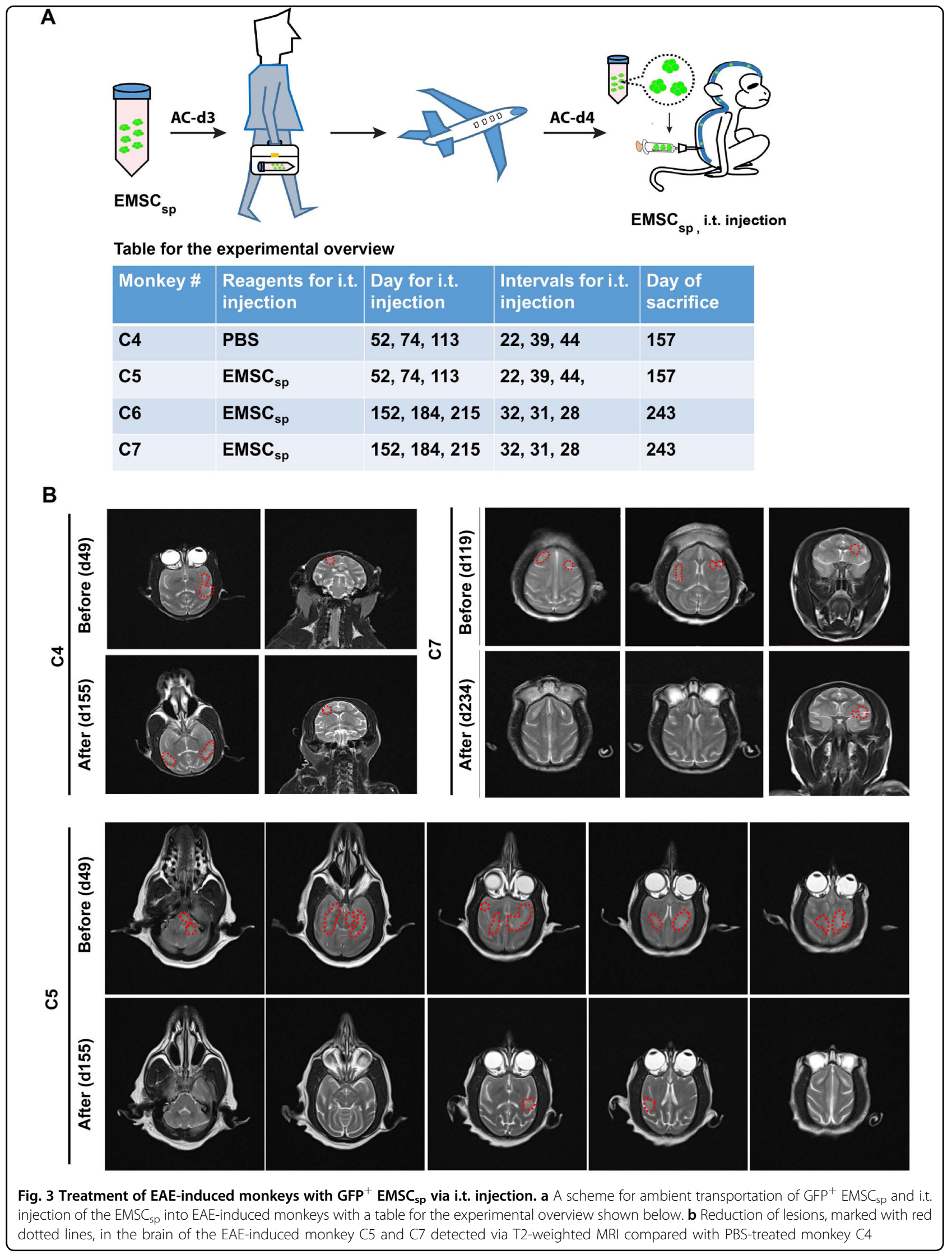




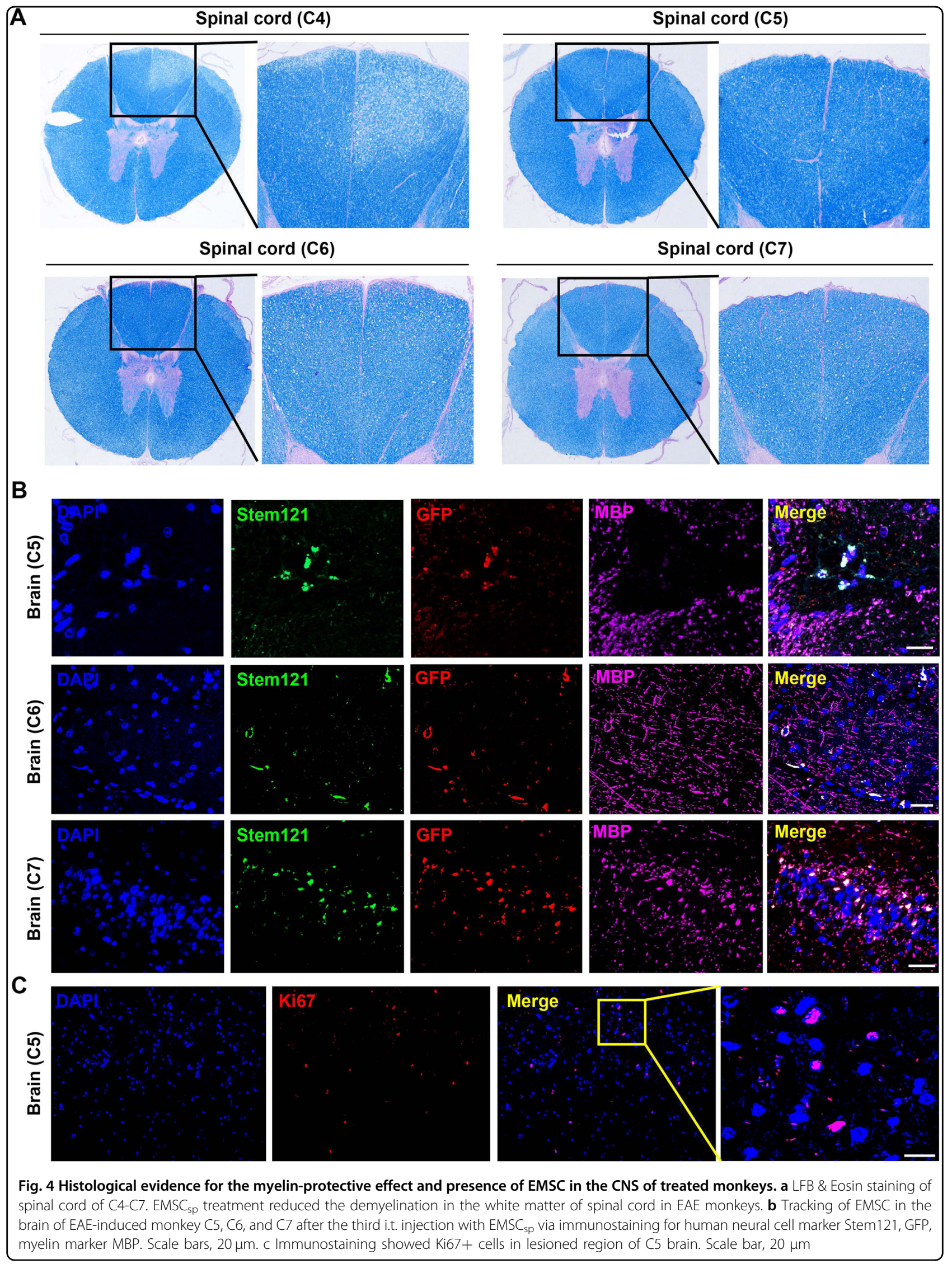


Meanwhile, we tested the levels of IL6 in the CSF and serum of the monkeys. IL6 plays a crucial role in pathogenesis of many inflammatory diseases in the $\mathrm{CNS}^{33}$. Indeed, IL6 in CSF dramatically increased in the EAE monkeys $\mathrm{C} 1$ and $\mathrm{C} 4-\mathrm{C} 6$, especially at the acute stage after the symptom onset (Fig. S4). Interestingly, after i.v. infusion, the IL6 in CSF slightly increased in both the control (C8) and EAE (C1) monkeys 4 days after the infusion (Fig. S4A). Nevertheless, the IL6 level in the CSF declined in the immunized monkeys no matter with $(\mathrm{C} 5-\mathrm{C} 7)$ or without (C4) i.t. injection of $\mathrm{EMSC}_{\mathrm{sp}}$ (Fig. S4B). These results indicate that (1) the IL6 level in the CSF increases in EAE monkey, (2) i.v. infusion of xenogeneic EMSC slightly stimulates IL6 secretion in the CNS, and (3) the IL6 level returns normal concurrent with the reduction or disappearance of the clinical score in the animals regardless EMSC treatments, (Fig. S1A).

\section{MRI tracking}

We conducted MRI examination on the treated animals following the i.t. injections. The lesions in the brain greatly reduced or disappeared in $\mathrm{C} 5$ at $\mathrm{d} 155$, compared with MRI images at $\mathrm{d} 49$ (Fig. 3b), which is consistent with the improvement of symptom scores (Fig. S1A). For C5, several lesions in the left side of the pons, the left hemisphere of the cerebella, and the subcortical lesion in the left frontal lobe vanished at 106 days after treatment, and multiple focal lesions in the subcortical white matter of bilateral temporal lobes decreased. Similar results were observed in C7 at d234 compared with MRI images at d119 (Fig. 3b). For C7, the subcortical lesions in bilateral frontal lobes and the subcortical lesion in the right temporal lobe disappeared $>100$ days after the treatment, and several small focal subcortical lesions in the left temporal lobe became smaller. On the contrary, the lesions of $\mathrm{C} 4$ treated with PBS showed no decrease in right frontal lobes but enlargement and newly formed lesions were found in both sides of the temporal lobe (Fig. 3b). These results showed that $\mathrm{EMSC}_{\mathrm{sp}}$ treatments reduce the lesion development in brain of EAE monkeys.

\section{Histological analyses for EMSC in the CNS of EAE monkeys}

To analyze the location, fate, and functionality of EMSC in the injected monkeys, we sacrificed the animals C5, C6, and $\mathrm{C7}$ after the $\mathrm{EMSC}_{\mathrm{sp}}$ treatments (Fig. 3a). LFB staining showed the $\mathrm{EMSC}_{\mathrm{sp}}$ treatment remarkably attenuate myelin loss in white matter of spinal cord in EMSC $_{\mathrm{sp}}$-treated monkeys (C5, C6, and C7) compared with PBS-treated monkey C4 (Fig. 4a). Moreover, demyelination, as well as active astrocytes, were observed in the lesion regions of $\mathrm{C} 4$ brain (Fig. S5A). $\mathrm{GFP}^{+}$cells were found around demyelinated lesions in the brain of C5 (Fig. 4b). GFP ${ }^{+}$cells were observed in the white matter of the brain of C6 and C7 (Fig. 4b). More interestingly, some $\mathrm{GFP}^{+}$cells were found also positive for Stem $121^{34}$ and MBP (Fig. 4b). These data suggest that EMSC can transdifferentiate into neural cells in the CNS. Moreover, $\mathrm{Ki}^{+} 7^{+}$cells were found in $\mathrm{C} 5$ brain indicating active regeneration of neural cells (Fig. 4c and Fig S5B). We did not find any $\mathrm{Ki}^{+} 7^{+}$cells in brain and spinal cord of $\mathrm{C} 4$. Besides, no Ki67 ${ }^{+}$cells were found to be positive for GFP. These results showed that our EMSC would not proliferate in monkey bodies.

\section{Transdifferentiation of EMSC into neural cells following culture in CSF in vitro}

Next, we attempted to recapitulate the in vivo transdifferentiation of EMSC into neural cells using an in vitro assay. AC-d2 $\mathrm{EMSC}_{\mathrm{sp}}$ were re-plated and spread in pooled CSF collected from the EAE monkeys (Fig. 5a). The cells in CSF gradually transformed into neural-like cells with outgrowing fibers from some cell bodies (Fig. 5b). Immunostaining shows that many of the CSF-cultured cells became positive for TUJ1 at d14 (Fig. 5c). The reverse transcriptase-PCR (RT-PCR) demonstrates that expression of neural marker genes SOX2, NESTIN, TUJ1, and MSI1 increased gradually during the CSF culture from $\mathrm{d} 0$ to $\mathrm{d} 7$ and $\mathrm{d} 14$, and expression of typical markers for oligodendrocytes NKX2.2, OLIGO2 and MOG also increased (Fig. 5d).

To elucidate the dynamic changes of the transcriptomic profile of EMSC cultured in CSF, we conducted microarray on $\mathrm{EMSC}, \mathrm{EMSC}_{\mathrm{sp}-\mathrm{AC} / \mathrm{d} 2}$, and $\mathrm{EMSC}_{\mathrm{sp}-\mathrm{AC} / \mathrm{d} 2}$ re-plated and

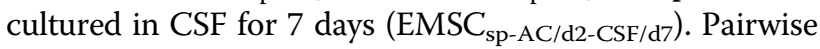
comparisons of the transcriptomic profiles of the three samples reveal a total of 7480 differentially expressed genes by more than twofold across all the comparisons (Fig. 6a). Particularly, when comparing $\mathrm{EMSC}_{\mathrm{sp}-\mathrm{AC} / \mathrm{d} 2-\mathrm{CSF} / \mathrm{d} 7}$ with EMSC, we obtained 1879 upregulated and 1456 downregulated genes (Table S1). Through gene function/ pathway enrichment analysis, many of the upregulated genes are associated with cell cycle, complex subunit and organelle organization, transportation and lipid metabolic process, neural trophic factors, neurogenesis, neuron differentiation, etc. On the other hand, many of the downregulated genes are related to regulation of cell communication, extracellular structure organization, angiogenesis, immune system process, MSC markers, etc. (Fig. 6b). These results support that CSF-cultured EMSC can transdifferentiate to neural lineages and produce neural trophic factors.

\section{Discussion}

Despite of the large body of literature on rodent EAE models, the monkey EAE model offers a rare opportunity for studies of MS in NHP. These models recapitulate many features of the demyelinating diseases, which allows researchers to elucidate the mechanism of the 


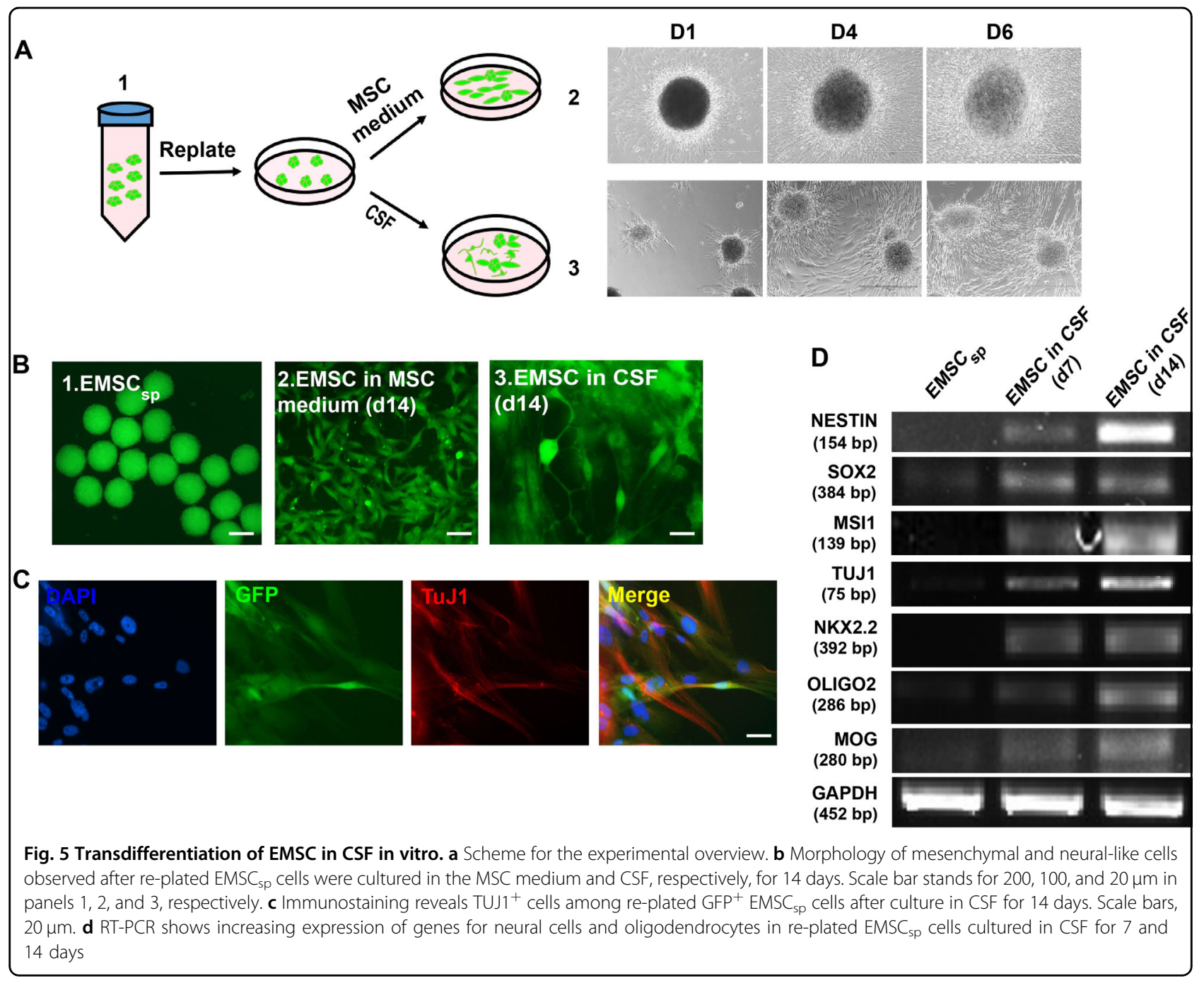

pathogenesis and test the efficacy of novel therapeutic approaches $^{5-9}$.

In this study, we induced EAE in seven cynomolgus monkeys. The success rate of EAE induction was 57 and $100 \%$ based on the symptom onset and MRI-detected brain lesions. The great variations in the symptom onset time and score are consistent with previous studies on monkey EAE models ${ }^{6-9}$. EAE has been induced in marmosets using recombinant human $\mathrm{MOG}_{1-125}$ plus incomplete Freund's adjuvant ${ }^{6,7,35}$. Compared with marmosets, cynomolgus has some advantages for EAE modeling as it is easy to draw CSF and blood for multiple times for its large body weight; vision problems and repeat relapsing-remitting courses resemble symptoms in MS patients ${ }^{36}$. Even in the symptomless cynomolgus monkeys following immunization, we observed lesions in brain, histological changes in the CNS, cytokine changes in the CSF, and alterations of leukocyte numbers in the peripheral blood. Based on the 3R principle for research on laboratory animals ${ }^{37}$, more than one experiment were conducted on some of the monkeys.

For treatment, spheropreserved $\mathrm{EMSC}_{\mathrm{sp}}$ were transported by flight from the laboratory in Macau to the animal facility in Kunming. Initially, we conducted i.v. injection of EMSC (dissociated from the ambiently transported $\mathrm{EMSC}_{\mathrm{sp}}$ ) on EAE-induced monkeys. No allergic symptoms or death happened in the injected animals, but GFP DNA declined rapidly within 7 days, implicating that EMSC reached the organs and then were rapidly eliminated. To achieve the highest concentration of EMSC in the CNS, we injected $\mathrm{EMSC}_{\mathrm{sp}}$ i.t. into the CNS of the EAEinduced monkeys. We observed therapeutic effects but no adverse effects during the entire experiments. We have previously shown that EMSC relieve murine EAE mainly via immunosuppression ${ }^{14}$. In addition, EMSC may encounter less immune rejection than other xenogeneic cells $^{14,26}$. It is unclear if immunosuppression also contributes to the efficacy of EMSC delivered directly into the 


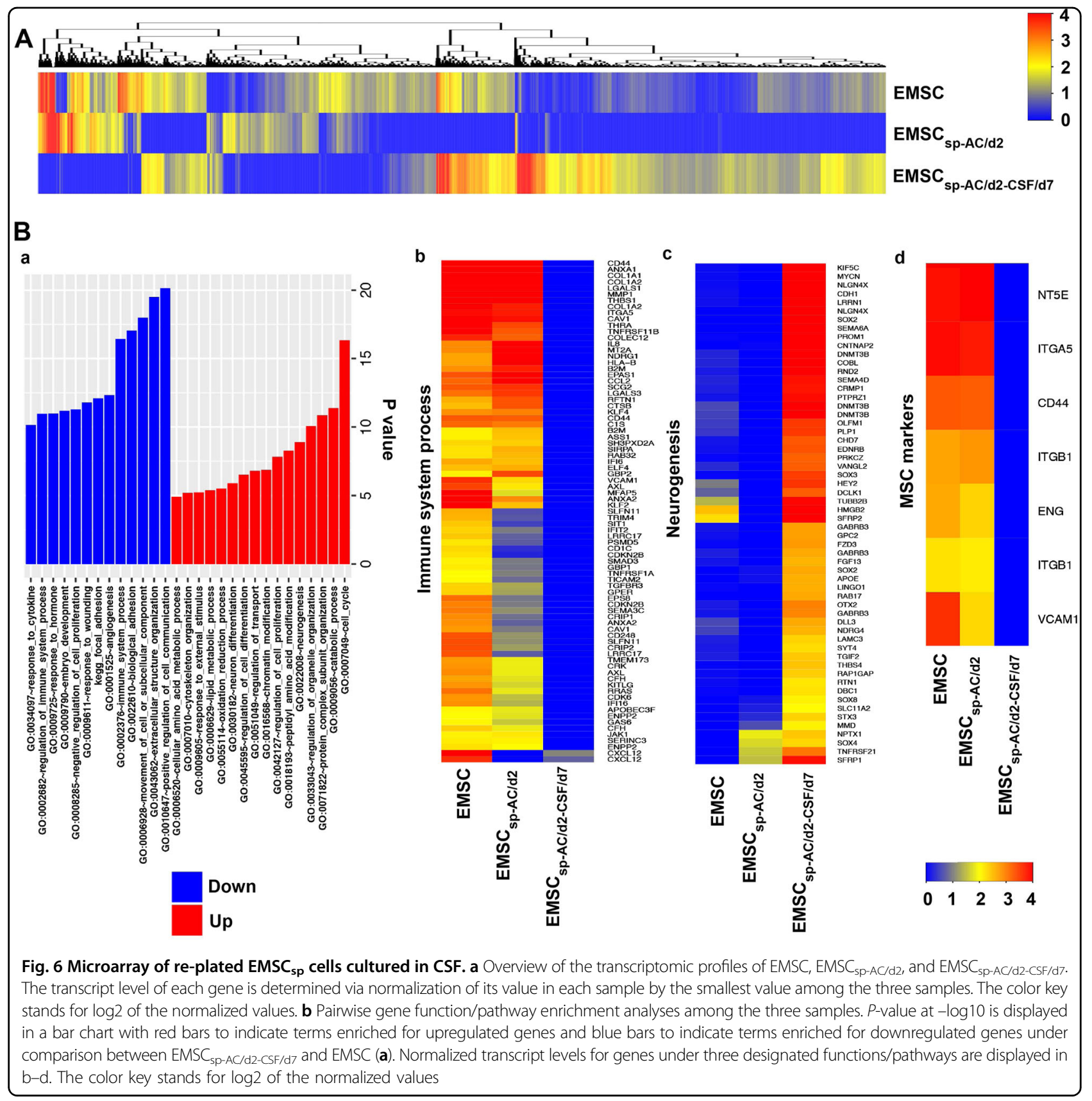

CNS in the monkey EAE model. Nevertheless, the MRI data, histological analyses of the CNS, and morphological and transcriptional analyses of CSF-cultured EMSC suggest that EMSC can reduce clinical score and brain lesions at least partially by migrating to the CNS and transdifferentiating into neurons and oligodendrocytes. The high levels of neural trophic factors secreted by EMSC cultured in CSF indicate that EMSC i.t. injected into the CNS may also promote endogenous neurogenesis and neuronal remyelination via these factors ${ }^{38-40}$.

There are some widely recognized concerns regarding cell therapies of hESC-derived cells, e.g., immunogenicity, tumorigenicity, inappropriate differentiation, and lack of organ specificity ${ }^{41}$. We found that $\mathrm{GFP}^{+}$human EMSC were present in the CNS of the EAE monkeys and some EMSC transdifferentiated into neural cells, indicating that EMSC might not encounter strong immune rejection in the CNS even after repeat i.t. injections. In addition, we found neither tumor in all the isolated organs nor inappropriate cell lineages differentiated from EMSC in the CNS about 100 days after the first $\mathrm{EMSC}_{\mathrm{sp}}$ treatment, based on rough macroscopic analyses (data not shown).

In this study, we only used EMSC derived from one $\mathrm{GFP}^{+} \mathrm{hESC}$ line (Envy) for tracking the cells in vivo. It 
would be ideal to use more cell lines and monkeys and observe for a longer period of time to consolidate our conclusions and identify inter-cell line variations. Nevertheless, this pilot study on monkeys, for the first time, demonstrates that $\mathrm{EMSC}_{\mathrm{sp}}$, following ambient transportation, can be delivered directly via i.t. injection into the subarachnoid cavity of the spinal cord, and promote recovery in a NHP model of EAE. Spheroidal formation and i.t. injection may both enhance the cell number and survival of EMSC in the CNS. Transdifferentiation of EMSC into as well as neural trophic effects of the transplanted cells may contribute to the efficacy of the cells as schemed in Fig. 7. The present study has extended our understanding of EMSC efficacy in the EAE models from mice to primates and may facilitate the translation of this potential cell therapy to humans.

\section{Experimental procedures}

\section{Animal care and ethic review}

Healthy female cynomolgus monkeys (Macaca fascicularis) at age of 8-9 years were selected for use in this study. All animals were housed at animal facility of Yunnan Key Laboratory of Primate Biomedical Research (LPBR). All of the animals were individually caged in an animal room with a 12 -h light and 12 -h darkness cycle. The temperature was maintained between $18^{\circ} \mathrm{C}$ and $26^{\circ} \mathrm{C}$ and with humidity from 40 to $70 \%$. Animals were fed twice per day with monkey chow. Fresh fruits and vegetables were supplemented once per day. All experimental procedures were reviewed and approved by the Institutional Animal Care and Use Committee of LPBR and were carried out in accordance with the Guide for the Care and Use of Laboratory Animals Eighth Edition. The IACUC approval number is LPBR20151201-01. The attending veterinarian followed ARRIVE guidelines to ensure appropriate handling, immobilization, sedation, analgesia, anesthesia, and euthanasia to ensure that proposed

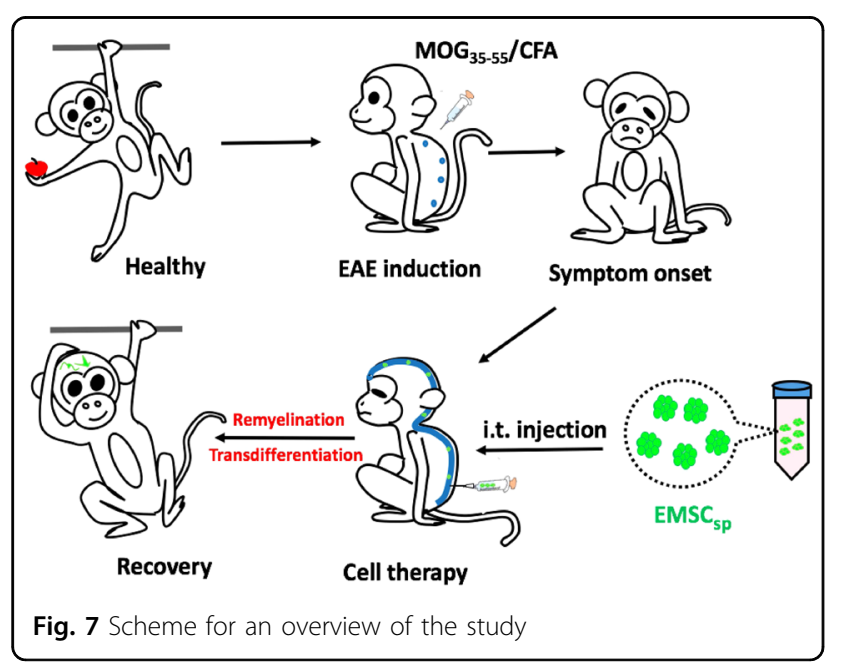

surgical procedures are appropriate. Abnormal postures, anorexia, vocalization, lethargy, and self-directed behaviors are examples of indicators, which are significant in evaluating postoperative pain or distress. Introduction of novel toys, cage location changes, or specialty foods (such as peanuts, raisins, or candies) are employed to moderate postoperative stress without compromising the scientific aspects of the protocol.

\section{EAE induction in cynomolgus monkeys}

EAE induction kit was purchased from Hook Laboratories for immunization of cynomolgus monkeys. $\mathrm{MOG}_{33-55}$ plus complete Freund's adjuvant emulsion was subcutaneously injected into four points in the dorsal skin at $100 \mu \mathrm{g} /$ point under systematic anesthesia. Anesthesia was performed via intramuscular injection of $5 \mathrm{mg} / \mathrm{kg}$ ketamine chloride (Fujian Gutian Pharmaceutical Inc.). The immunization was boosted once or twice until symptom onset in the animals (Fig. 1A). The symptoms of the monkeys were scored daily by two experienced observers using a standard scoring system: ${ }^{6,8} 0=$ no clinical signs; $0.5=$ loss of appetite and apathy; $1=$ lethargy, anorexia, and tremor; $2=$ ataxia and optic problems; $2.5=$ paraperesis and sensory loss; $3=$ hemiplegia or paraplegia; $4=$ complete paralysis (quadriplegia); $5=$ moribund. For ethical reason, a monkey must be humanly terminated when it reaches symptom score $\geq 3.0$.

\section{Cell preparation, transportation, and injection}

EMSC were derived from the Envy hESC line ${ }^{31}$ using the derivation and verification methods as we reported ${ }^{27}$. The resultant EMSC were cultured in a MSC medium including minium essential medium alpha supplemented with $20 \%$ fetal bovine serum, L-glutamine, and $1 \times$ nonessential amino acids (Gibco, Grand Island, NY) until 80\% confluence $^{26}$. EMSC were then dissociated and cultured in a $37{ }^{\circ} \mathrm{C}$ incubator for 2 days to form spheres with 15,000 cells each and $200 \mu \mathrm{m}$ in diameter using the hanging-drop method ${ }^{30}$. The spheroids were collected in a sealed 15-ml tube filled with the MSC medium, which was placed under ambient conditions (at room temperature) for 3 days (AC-d3) and then transported by flight to the animal facility in Kunming (about $2000 \mathrm{~km}$ away). The spheres were either dissociated for i.v. injection $\left(1 \times 10^{7}\right.$ cells $/ \mathrm{kg}$, all the cells suspended in a total of $50-\mathrm{ml}$ PBS) or directly used for i.t. injection into the monkeys with the number of spheres equivalent to $2 \times 10^{7}$ cells/monkey. The cell viability was determined using AO/PI (Cellometer), and apoptotic cells were analyzed using the Annexin-V/PI kit.

Before i.t. injection, the monkeys were anesthetized with ketamine as described above, and their dorsal skin was shaved and sterilized following standard aseptic procedures. A spinal syringe was inserted into the subarachnoid 
cavity of the spinal cord, and 2-ml CSF was released from the syringe to leave space for injection of the sphere suspension to avoid pressure increase in the CNS. The CSF was collected, filtered for sterilization, aliquoted, and frozen for further experiments. A 2-ml sphere suspension or PBS (as the negative control) was then slowly injected into the subarachnoid cavity. After the injection, the animals were returned to their cages for recovery and monitored for their response to the injection such as hyperspasmia and fever.

\section{Cytometric bead array for cytokine measurement}

CSF collected from monkeys were assayed for levels of multiple cytokines using the Cytometric Bead Array Nonhuman primate cytokine kit (BD Biosciences) according to the manufacturer's instructions. A series of cytokines were detected on the BD Accuri ${ }^{\mathrm{TM}} 6$ flow cytometry. The concentration of the cytokine is calculated based on standard curves generated from serial dilutions of cytokine standards provided by the manufacture.

\section{Magnetic resonance imaging}

Upon the symptom onset and following the EMSC treatment, MRI was conducted to monitor lesions in the brain of the animals. Monkeys were anesthetized as described above, and their brain MRI scans were performed before and after the EMSC treatments on a 1.5-T MAGNETOM ESSENZA unit (Siemens). The brains were imaged with axial, coronary, and sagittal sequences using an identical field of view $(240 \mathrm{~mm} \times 210 \mathrm{~mm})$. The slice thickness was $3 \mathrm{~mm}$. T2-weighted turbo spin-echo (repetition time (TR)/echo time (TE) was 3800-4500 ms/97-103 ms, T1-weighted spin-echo TR/TE was 550 $\mathrm{ms} / 11 \mathrm{~ms}$, and fluid-attenuated inversion recovery TR/TE was $9000 \mathrm{~ms} / 87 \mathrm{~ms}$. All images were reviewed with a DICOM Viewer (RadiAnt) and analyzed by experienced neurologists who were blinded to the treatment statuses of the monkeys.

\section{Euthanasia of monkeys and tissue sampling}

Upon termination of an experiment, monkey euthanasia was conducted with sodium pentobarbital $(100 \mathrm{mg} / \mathrm{kg})$ via i.v injection, according to the criteria of the guide. Technicians were allowed to perform euthanasia only after they had been trained in the proper techniques and humane considerations. The death of animals was confirmed based on the appearance of irreversible coma, completely lost response to outside stimuli, and disappearance of spontaneous breathing and cranial nerve reflex such as pupil reflex, corneal reflection, and swallowing reflex. After that, the animals were perfused i.v. with $1.5-\mathrm{L}$ saline solution to flush out all the blood. Fresh tissues were dissected from the major organs including the brain (the frontal lobe), spinal cord (the cervical vertebrae), lungs, heart, liver, spleen, pancreas, kidneys, bladder, uterus, inguinal lymph nodes, and skin, and immediately frozen in liquid nitrogen for gDNA and RNA isolation, and the left tissues were fixed with $4 \%$ paraformaldehyde for 1 week. Fixed tissues were washed twice with PBS and transferred to $70 \%$ ethanol for further storage and section.

\section{Histological analyses}

The brain and spinal cord of the monkeys were dehydrated and paraffin embedded using the Tissue Processor and EG1150 Embedding Centre (Leica) following the standard procedures. Paraffinized tissues were cut into thin slices at $6 \mu \mathrm{m}$ on a RM2235 Microtome (Leica). Sections were processed for deparaffinization and rehydration manually with xylene and gradient ethanol. For histochemical staining, Luxol Fast Blue and eosin staining were used to evaluate demyelination in the brain and spinal cord.

For immunostaining, deparaffinized tissue sections were placed at a sub-boiling temperature in a microwave for 15 min for epitope retrieval. To prevent nonspecific binding, we blocked each section with $3 \%$ bovine serum albumin at room temperature for $1 \mathrm{~h}$ in a black humid chamber. Antibodies used in this study were listed in Table S2. Each section was incubated with a desired primary antibody at $4{ }^{\circ} \mathrm{C}$ overnight followed by washing and staining with a corresponding secondary antibody at room temperature for $1 \mathrm{~h}$, and the nucleus counterstained with 4,6-diamidino-2-phenylindole. For the immunohistochemical procedures, a 3,3'-diaminobenzidine (DAB) kit (Tiangen) was used following user manual after washing the secondary antibody. For observation of the histological structures, the sections were stained with Luxol Fast Blue and eosin staining following standard procedures. All images were captured using Carl Zeiss Axio Observer (Zeiss).

\section{Genomic DNA and RNA isolation and RT-qPCR}

A variety of tissues were isolated from the monkeys i.v. injected with $\mathrm{GFP}^{+}$EMSC and treated with a tissue lysis buffer (Tiangen) supplemental with proteinase $\mathrm{K}$ at $56^{\circ} \mathrm{C}$ overnight. In all, 200-ng gDNA isolated from the samples through phenol/chloroform extraction was used for detection of the level of the GFP gene (presented as ng/ $\mu \mathrm{g}$ gDNA) using primer listed in Table S3. Total RNA was extracted from the monkey tissues by using Trizol reagent (Life Technology) and complementary DNA (cDNA) was generated through RT using PrimeScript RT Reagent kit (Clontech). Primers for specific genes were listed in Table S3. Regular RT-PCR reactions were carried out withTaq5 $\times$ Master Mix (New Egland Biolabs) under following conditions: an initial denaturation at $95^{\circ} \mathrm{C}$ for $30 \mathrm{~s}$; followed by 28 cycles of $30 \mathrm{~s}$, denaturation at $95^{\circ} \mathrm{C}$ for 20 $\mathrm{s}$, annealing at $53^{\circ} \mathrm{C}$ for $30 \mathrm{~s}$ with a final extension at $68^{\circ} \mathrm{C}$ 
for $10 \mathrm{~min}$. qPCR reactions were conducted using $\mathrm{iTaq}^{\mathrm{TM}}$ Universal SYBR Green Supermix (Bio-Rad), and detected with the CFX96TM Real-Time PCR Detection System (Bio-Rad). qPCR was performed under following conditions: an initial denaturation at $95^{\circ} \mathrm{C}$ for $5 \mathrm{~s}$ followed by 39 cycles of 5 -s denaturation at $95^{\circ} \mathrm{C}$ for 30 -s annealing at $60^{\circ} \mathrm{C}$, melt curve analysis by 0.5 increments at $5 \mathrm{~s} / \mathrm{step}$ from 65 to $95^{\circ} \mathrm{C}$. Final incubation was at $95^{\circ} \mathrm{C}$ for $30 \mathrm{~s}$ for polymerase activation and DNA denaturation.

\section{Microarray analysis of EMSC cultured in CSF}

RNA was extracted from cells harvested under various conditions by using RNAeasy mini kit (Qiagen) and cDNA libraries were synthesized and processed by using HumanHT-12 v4 Expression Bead Chip. Further analysis was performed using the Bead Studio Data Analysis software (Illumina) and Excel. The microarray dataset was normalized and filtered to remove probes with detection $P$-value $>0.01$ in at least $50 \%$ of the samples. Two normalization methods were utilized to analyze the dataset on the basis of their biological relevance. First, the average probe intensity of all biological and technical replicates was used to compare the test and control groups for their gene expression profiles. Second, the probe intensity of analyzed genes in the control sample EMSC was used as a baseline to identify the expression of the genes in the test samples. Pathway enrichment was assessed using a hypergeometric test against gene sets collected from Msigdb covering pathways in the Kyoto Encyclopedia of Genes and Genomes (KEGG), Biocarta, Reactome, and the Gene Ontology (GO) terms. In order to control the false discovery rate, we set statistical significance at $P=$ 0.001 as the cutoff for a pathway enrichment test. The original data from microarray were deposited in the GEO repository (accession \# GSE107145).

\section{Data analysis}

All flow cytometry data were analyzed and generated by FlowJo 7.6. All figures were prepared using GraphPad Prism.

\section{Acknowledgements}

We appreciate the excellent services provided for this study by the staff of the Yunnan Key Laboratory of Primate Biomedical Research, and the Genomics Core, Single Cell and Gene Expression Analysis Core, Histopathology Core, and Metabolics Core of Faculty of Health Sciences, University of Macau. This work was supported by University of Macau Research Committee funds MYRG 201500169-FHS to R.-H.X., National Key Research and Development Program of China (2016YFA0101400) and National and Provincial Natural Science Foundation of China (U1302227) to Y.N., National Natural Science Foundation of China (31660346) to W.S., Macau Science and Technology Development Fund (FDCT) 128-2014-A3, 028/2015/A1, and 095/2017/A2, MYRG 2016-00070FHS and 2017-00124-FHS to R.-H.X.

\footnotetext{
Author details

${ }^{1}$ Faculty of Health Sciences, University of Macau, Taipa, Macau, China. ${ }^{2}$ Yunnan Key Laboratory of Primate Biomedical Research, Institute of Primate Translational Medicine, Kunming University of Science and Technology, Kunming, Yunnan, China. ${ }^{3}$ Sun Yat-sen Memorial Hospital, Sun Yat-sen
}

University, Guangzhou, Guangdong, China. ${ }^{4}$ Key Laboratory of Symbolic Computation and Knowledge Engineering of Ministry of Education, College of Computer Science and Technology, Jilin University, Changchun, Jilin, China

\section{Authors' contributions}

L.Y., B.J., Y.N., W.J., R.H.X. and W.S. conceived and designed the experiments. L.Y., Y.N., B.J. and W.S. established the EAE monkeys. L.Y., B.J. and E.L. prepared and transported the cells. W.S., Y.N., Y.Y. and S.C. performed cell injections. Y.Y., Y.D. and S.C. monitored and scored the symptoms of the monkeys. H.W. analyzed the MRI images. H.S. assisted the CSF experiments and gene expression analyses. L.Y., B.J., G.C., Y.N. and W.S. performed the other experiments and assembled the data. L.Y., W.S., B.J. and R.H.X. wrote the manuscript. All authors revised and gave the final approval of the manuscript.

\section{Conflict of interest}

R.-H.X. is a founder of ImStem Biotechnology, Inc., a stem cell company. He declares competing financial interests. The remaining authors declare that they have no conflict of interest.

\section{Publisher's note}

Springer Nature remains neutral with regard to jurisdictional claims in published maps and institutional affiliations.

The online version of this article (https://doi.org/10.1038/s41420-018-0091-0) contains supplementary material, which is available to authorized users.

Received: 30 May 2018 Revised: 27 June 2018 Accepted: 5 July 2018 Published online: 20 August 2018

\section{References}

1. Dendrou, C. A., Fugger, L. \& Friese, M. A. Immunopathology of multiple sclerosis. Nat. Rev. Immunol. 15, 545-558 (2015).

2. Jagessar, S. A. et al. Unravelling the T-cell-mediated autoimmune attack on CNS myelin in a new primate EAE model induced with MOG34-56 peptide in incomplete adjuvant. Eur. J. Immunol. 42, 217-227 (2012).

3. Kap, Y. S. et al. Effects of early IL-17A neutralization on disease induction in a primate model of experimental autoimmune encephalomyelitis. J. Neuroimmune. Pharmacol. 6, 341-353 (2011).

4. Hemmer, B., Kerschensteiner, M. \& Korn, T. Role of the innate and adaptive immune responses in the course of multiple sclerosis. Lancet Neurol. 14, 406-419 (2015).

5. Constantinescu, C. S., Farooqi, N., O'Brien, K. \& Gran, B. Experimental autoimmune encephalomyelitis (EAE) as a model for multiple sclerosis (MS). Br. J. Pharmacol. 164, 1079-1106 (2011).

6. Haanstra, K. G. et al. Induction of experimental autoimmune encephalomyelitis with recombinant human myelin oligodendrocyte glycoprotein in incomplete Freund's adjuvant in three non-human primate species. J. Neuroimmune. Pharmacol. 8, 1251-1264 (2013).

7. Jagessar, S. A. et al. Immune profile of an atypical EAE model in marmoset monkeys immunized with recombinant human myelin oligodendrocyte glycoprotein in incomplete Freund's adjuvant. J. Neuroinflamm. 12, 169 (2015).

8. Kap, Y. S. et al. Fast progression of recombinant human myelin/oligodendrocyte glycoprotein (MOG)-induced experimental autoimmune encephalomyelitis in marmosets is associated with the activation of MOG34-56-specific cytotoxic T cells. J. Immunol. 180, 1326-1337 (2008).

9. $t$ Hart, B. A. et al. Suppression of ongoing disease in a nonhuman primate model of multiple sclerosis by a human-anti-human IL-12p40 antibody. J. Immunol. 175, 4761-4768 (2005).

10. Rivera, F. J. \& Aigner, L. Adult mesenchymal stem cell therapy for myelin repair in multiple sclerosis. Biol. Res. 45, 257-268 (2012).

11. Rice, C. M., Kemp, K. Wilkins, A. \& Scolding, N. J. Cell therapy for multiple sclerosis: an evolving concept with implications for other neurodegenerative diseases. Lancet 382, 1204-1213 (2013).

12. Thiruvalluvan, A. et al. Survival and functionality of human induced pluripotent stem cell-derived oligodendrocytes in a nonhuman primate model for multiple Ssclerosis. Stem Cells Transl. Med. 5, 1550-1561 (2016). 
13. Kim, H. et al. Immunomodulation by transplanted human embryonic stem cell-derived oligodendroglial progenitors in experimental autoimmune encephalomyelitis. Stem Cells 30, 2820-2829 (2012).

14. Wang, X. et al. Human ESC-derived MSCs outperform bone marrow MSCs in the treatment of an EAE model of multiple sclerosis. Stem Cell Rep. 3, 115-130 (2014).

15. Wang, $X$. et al. Immune modulatory mesenchymal stem cells derived from human embryonic stem cells through a trophoblast-like stage. Stem Cells $\mathbf{3 4}$ 380-391 (2016).

16. Donega, M., Giusto, E., Cossetti, C., Schaeffer, J. \& Pluchino, S. Systemic injection of neural stem/progenitor cells in mice with chronic EAE. J .Vis. Exp. https://doi. org/10.3791/51154 (2014).

17. Mohyeddin Bonab, M. et al. Does mesenchymal stem cell therapy help multiple sclerosis patients? Report of a pilot study. Iran. J. Immunol. 4, 50-57 (2007).

18. Mohyeddin Bonab, M. et al. Evaluation of cytokines in multiple sclerosis patients treated with mesenchymal stem cells. Arch. Med. Res. 44, 266-272 (2013).

19. Connick, P. et al. Autologous mesenchymal stem cells for the treatment of secondary progressive multiple sclerosis: an open-label phase $2 a$ proof-ofconcept study. Lancet Neurol. 11, 150-156 (2012).

20. Yamout, B. et al. Bone marrow mesenchymal stem cell transplantation in patients with multiple sclerosis: a pilot study. J. Neuroimmunol. 227, 185-189 (2010).

21. Karussis, D. et al. Safety and immunological effects of mesenchymal stem cell transplantation in patients with multiple sclerosis and amyotrophic lateral sclerosis. Arch. Neurol. 67, 1187-1194 (2010).

22. Azari, M. F. et al. Mesenchymal stem cells for treatment of CNS injury. Curr. Neuropharmacol. 8, 316-323 (2010)

23. Ankrum, J. A., Ong, J. F. \& Karp, J. M. Mesenchymal stem cells: immune evasive, not immune privileged. Nat. Biotechnol. 32, 252-260 (2014).

24. Yan, L., Zheng, D. \& Xu, R.-H. Critical role of tumor necrosis factor signaling in mesenchymal stem cell-based therapy for autoimmune and inflammatory diseases. Front. Immunol. 9, 1658 (2018).

25. Le Blanc, K. \& Mougiakakos, D. Multipotent mesenchymal stromal cells and the innate immune system. Nat. Rev. Immunol. 12, 383-396 (2012).

26. Wang, $X$. et al. Immune modulatory mesenchymal stem cells derived from human embryonic stem cells through a trophoblast-like stage. Stem Cells https://doi.org/10.1002/stem.2242 (2015).
27. Yan, L. et al. Scalable generation of mesenchymal stem cells from human embryonic stem cells in 3D. Int. J. Biol. Sci. 14, 1196-1210 (2018).

28. Jung, Y., Bauer, G. \& Nolta, J. A. Concise review: induced pluripotent stem cellderived mesenchymal stem cells: progress toward safe clinical products. Stem Cells 30, 42-47 (2012).

29. Xie, C., Liu, Y. Q., Guan, Y. T. \& Zhang, G. X. Induced stem cells as a novel multiple sclerosis therapy. Curr. Stem. Cell Res. Ther. 11, 313-320 (2016).

30. Jiang, B. et al. Spheroidal formation preserves human stem cells for prolonged time under ambient conditions for facile storage and transportation. Biomaterials 133, 275-286 (2017).

31. Costa, M. et al. The hESC Ine envy expresses high levels of GFP in all differentiated progeny. Nat. Methods 2, 259-260 (2005).

32. Ezquer, F. et al. Intravenous administration of anti-inflammatory mesenchyma stem cell spheroids reduces chronic alcohol intake and abolishes bingedrinking. Sci. Rep. 8, 4325 (2018).

33. Erta, M., Quintana, A. \& Hidalgo, J. Interleukin-6, a major cytokine in the central nervous system. Int. J. Biol. Sci. 8, 1254-1266 (2012).

34. Salazar, D. L., Uchida, N., Hamers, F. P., Cummings, B. J. \& Anderson, A. J. Human neural stem cells differentiate and promote locomotor recovery in an early chronic spinal cord injury NOD-scid mouse model. PLoS ONE 5, e12272 (2010).

35. $t$ Hart, B. A. et al. The primate EAE model points at EBV-infected B cells as a preferential therapy target in multiple sclerosis. Front. Immunol. 4, 145 (2013).

36. Nam, K. H. Experimental autoimmune encephalomyelitis in cynomolgus monkeys. J. Vet. Sci. 1, 127-131 (2000).

37. t Hart, B. A. Primate autoimmune disease models; lost for translation? Clin Transl. Immunol. 5, e122 (2016).

38. Cristofanilli, M. et al. Mesenchymal stem cells enhance the engraftment and myelinating ability of allogeneic oligodendrocyte progenitors in dysmyelinated mice. Stem Cells Dev. 20, 2065-2076 (2011).

39. Jeong, C. H. et al. Mesenchymal stem cells expressing brain-derived neurotrophic factor enhance endogenous neurogenesis in an ischemic stroke model. Biomed. Res. Int. 2014, 129145 (2014).

40. Kassis, l. et al. Neuroprotection and immunomodulation with mesenchymal stem cells in chronic experimental autoimmune encephalomyelitis. Arch. Neurol. 65, 753-761 (2008).

41. Zheng, D., Wang, X. \& Xu, R. H. Concise review: one stone for multiple birds: generating universally compatible human embryonic stem cells. Stem Cells $\mathbf{3 4}$, 2269-2275 (2016) 